\title{
A RE-AIM Evaluation of a multi-modal real-world intervention for dementia risk reduction
}

\section{Christine Stirling ( $\square$ Christine.Stirling@utas.edu.au )}

University of Tasmania https://orcid.org/0000-0003-2723-8302

\section{Helga Merl}

The University of Newcastle - Newcastle City Campus

Indra Arunachalam

Integratedliving

\section{Ashley Turner}

The University of Sydney

\section{Carolyn King}

University of Tasmania

\section{Research article}

Keywords: Health behaviour, Independent living, Loneliness, Nursing, Preventative Health

Posted Date: July 3rd, 2019

DOI: https://doi.org/10.21203/rs.2.10970/v1

License: (c) (i) This work is licensed under a Creative Commons Attribution 4.0 International License. Read Full License 


\section{Abstract}

Background This implementation study evaluated a novel community-based Memory Wellness Program targeting dementia risk reduction through increasing health knowledge and health promoting behaviours in older adults. The nurse-led eight-week program involved the following behaviour change strategies: goal-setting, education, group activity, and introduction to the use of iPads and Misfit activity trackers. The multi-model program was delivered in a realworld setting using pop-up clinics run by a Registered Nurse, across 18 different sites. Methods Using a quasi-experimental mixed methods design, and a REAIM framework, the study evaluated the reach, effectiveness, adoption, implementation and maintenance of the MWP. A total of 179 older adults aged 65 years and over participated in the evaluation across eighteen locations in three states and a territory of Australia. Paired t-tests were carried out on all pretestposttest clinical data. In-depth interviews were conducted with seventeen participants and staff. Results Engagement in the program was associated with improved cognition, lowered BP and stress and increased engagement with technology. There was a trend towards reduced feelings of loneliness. Participants' main motivations for undertaking the program were to improve memory, meet new people and improve technology use and computer literacy, with most participants reporting that these goals had been met through the program. The program was effective across multiple sites demonstrating it can successfully be implemented in different contexts and that the range of behaviour change techniques suit a useful multi-modal program. Conclusions This evaluation of a community-based Memory Wellness Program demonstrated increased cognitive function in older adults presenting with concerns about their cognition and memory. The statistically significant results and medium to large effect sizes suggest that further research is warranted to assess the efficacy of multi-modal community-based programs for improving memory and mental health with a focus on dementia risk reduction in older adults.

\section{Background And Objectives}

The numbers of people with dementia are growing rapidly. In the absence of disease-modifying therapeutics, a large body of research has focussed efforts on identifying early intervention strategies aimed at slowing disease progression or potentially reversing levels of cognitive decline for those who are at risk of developing dementia (1). As dementia is now increasingly recognised at an earlier stage, and the numbers of people with prodromal stages of dementia increases, there is an increasingly strong voice from people either at risk of dementia or living with dementia for such interventions. The potential to prevent millions of cases of dementia with concomitant benefits to individuals and society provide a strong rationale for prevention and early intervention efforts (2).

There is increasing evidence that multi-modal risk factor modification may provide the most benefits in efforts to delay or prevent the onset of dementia (3-5). Adopting a healthy lifestyle with physical activity, good nutrition, and cognitive and social activities appear to be key to risk modification for dementia (2). In response to increasing evidence of risk modification benefits integratedliving Australia developed the Memory Wellness Program (MWP), which was funded nationally by the Australian Government Department of Health. The program has three key elements, an 8-week group-based health promotion 
course, a 4-week period of self-directed activities, and a Registered Nurse (RN) clinical assessment that was given at the beginning and end of the program designed to screen for conditions such as hypertension, dementia, and diabetes.

The combination of healthy lifestyle factors that are known to help prevent both cardiovascular disease and diabetes, appear likely to have benefits for brain health and cognition. Physical activity has widely proven health benefits, and is linked to brain health (2) with a strong level of evidence as a modifiable cognitive decline risk factor, but only a moderate level of evidence for dementia. While the strongest evidence exists for more vigorous exercise, epidemiology research suggests even modest exercise for seniors commencing an activity program improves cognitive function (6).

There is a moderate level of evidence that a Mediterranean diet decreases the risk of cognitive decline and a lower level of evidence that it decreases the risk of dementia (3). While many studies have looked at the efficacy of particular dietary elements, only omega-3 and flavonoids have shown evidence of possible beneficial effects on cognition $(4,7)$. Overall factors that improve cardiovascular health (particularly hypertension) have a strong association with lowering dementia risk $(3,4)$.

The MWP targeted both social and cognitive activity as well as the traditional health behaviors of physical activity and diet. The Finnish Geriatric Intervention Study to Prevent Cognitive Impairment and Disability (Finger) demonstrated improved cognition from a two-year multi-modal intervention targeting physical activity, diet, cognitive training, management of cardiovascular risk factors and social activity (5). This intensive trial though was costly and high intensity. Other programs have demonstrated improved cognition and activity from less intensive interventions (8-10) and the MWP aimed to deliver a sustainable program that could reach even rural populations.

There has been a strong focus on testing the benefits of cognitive training programs with a systematic review showing benefits for numerous aspects of cognition and wellbeing, including memory and mood (3). However, while the outcomes are clear, the mechanism of action is not and there is a growing recognition that the benefits could be provided from cognitive stimulation in the form of social and other activities, not just cognitive training that improves cognition in older people with dementia. A metaanalysis of cognitive stimulation in older people with dementia identified that programs that engaged participants in activities and discussions aimed at improving cognitive and social functioning did improve cognition (11). There has been considerable research suggesting that positive social integration is associated with a lower risk of dementia (3) and evidence that increased levels of social networks and social engagement are associated with reduced cognitive decline $(12,13)$. More recently loneliness (people's subjective experience of poor-quality social relationships) and not social isolation (living arrangements and social networks) has been found to be positively associated with an increased risk of dementia (14). A recent study trialed the use of internet based social media as a form of social interaction and found short-term improvements in cognition (15). The MWP provided clients with both tablet technology and activity monitoring wrist bands plus training, with the expectation that learning to 
use the technology and using relevant applications would provide them clients with both cognitive stimulation and social interaction benefits, along with some health promotion reinforcement.

Goal-setting and group work were also fundamental to encouraging both behavior change and social activity in the MWP clients. Goal setting is increasingly seen as an effective means of ensuring that interventions are patient-centred and provide intrinsic motivation for behaviour change $(16,17)$. The most positive results from goal setting appear to occur in programs where the goals are made public to an audience beyond the individual or set by a group together $(17,18)$. Wearable tracking technologies has also been demonstrated to increase people's activity levels, with goal setting indicated as part of their effectiveness (19). Conner et al. (20) found that clear goal priority resulted in stronger intentions and behaviour changes, compared to goal conflict.

This paper reports a real-world evaluation of the MWP, meaning that the evaluators had no control over the intervention or context. This approach to research aims to decrease the science practice gap and the 17 year implementation timeframe for uptake by prioritizing evidence that is representative of 'the realities in which end users live and work' (21: 156). For community based primary care interventions the impact of context and adaptability of interventions are vital to uptake and success. For the MWP intervention some might argue that more trials are needed to understand how the individual risk factors impact on dementia incidence and the best dose for targeted interventions, yet this type of evidence has little chance of impacting practice because neither the setting nor the participants adequately reflect real world settings. For example, most dementia research does not study those over 85 years of age, often recruiting from specialists clinics where people with dementia are often younger and with rarer conditions (22). This research meets the need for evidence where the major effort focuses on prevention to 'the greatest extent possible by early intervention' situated in the reality of 'real populations' that acknowledge dementia as largely a geriatric syndrome (23). This paper describes the real-world RE-AIM evaluation of the multi-modal MWP, which involved participants representative of the general population across diverse settings, and delivered a cost-effective intervention targeting improved brain health.

\section{Methods}

This mixed methods evaluation used the five elements of the RE-AIM framework (reach, effectiveness, adoption, implementation and maintenance) to assess a real-world multi-modal program aimed a decreasing cognitive decline risk factors. Glasgow (24) developed the RE-AIM framework to improve health promotion evaluations to include broader population-based concerns. By including elements such as population reach, program uptake and program sustainability the RE-AIM framework addresses far more than the common effectiveness and implementation scope of most evaluations.

\section{Participant Recruitment}

The evaluation invited all MWP clients to participate in the evaluation, with 200 clients given an information sheet by integratedliving's RNs when they attended the first session of the MWP. Of these, $90 \%$ consented in writing for their de-identified program records to be accessed for the evaluation, 
resulting in 170 participants from 18 pop-up clinic sites across four Australian states and territory. For the Tasmanian arm, a more comprehensive data collection required separate ethics processes. MWP clients were given the information sheet and requested to contact the research team member if they wished to participate. Sixty-seven participants were recruited from six Tasmanian MWP groups into the study over a one-year period, with four withdrawals before baseline data collection and only two Tasmanian MWP participants refusing consent. Thirteen Tasmanian clients were selected for interview, with the sampling framework based on maximising diversity of age, gender, and program location. The three Tasmanian RNs delivering the program and the program manager were also interviewed.

\section{Data collection}

The study collected pre and post clinical information about blood pressure (BP), weight, body mass index (BMI) blood glucose level (BGL), Addenbrooke's Cognitive Examination III (ACE-III), and depression, anxiety and stress scales (DASS 21). The aim was to collect this clinical data from all participants enrolled into the MWP over 2 years ( 200). However, while $90 \%$ of clients were consented into the study, the RNs running the clinics did not follow the pre and post clinical measure aspect of the MWP closely, which resulted in large gaps in the data collection. The ACE-III had the best coverage with 170 pre/post sets from 18 groups along with self-selected goals.

Additional data was collected across six groups in Tasmania, including the short form of the UCLA Loneliness Scale (ULS-6; (25)), The Technology/Internet Use Questionnaire (TIU; (26)) and the Physical Activity Stages of Change measure (PA-SoC (27)).

ACE-III is a well validated screening test for dementia (28). The MWP used AceMobile (which is ACE-III delivered via $\mathrm{iPad}$ ), which has demonstrated a $85-93 \%$ reduction in administrative error rates compared to paper based delivery (29). The ULS-6 is a 6-item questionnaire measuring subjective feelings of loneliness and social isolation. Psychometric analysis of this measurement tool in a sample of older adults revealed that the ULS-6 provides a brief yet psychometrically sound measure of loneliness that is appropriate for use among older adults (25). The TIU (adapted from (26)) is an 8-item questionnaire about the use of mobile and computer technologies, as well as internet-based activities. The TIU was included to measure changes in clients' use of computer and internet technologies across the span of the program. The PA-SoC is based on the Transtheoretical Model of Behaviour Change and measures one's intentions to engage in physical activity in 4-items. This data will indicate whether clients' intentions to engage in physical activity changes following MWP education sessions.

The Mini Nutritional Assessment was undertaken for initial clients but was dropped as part of the data collection as it was found not to be sensitive enough to measure changes in eating behaviour pre/post MWP. No other suitable tools were identified.

\section{Qualitative}


The interview schedule for both participants and nurses asked about motivation for joining the program, any memory concerns, technology use, and whether key elements of the program were beneficial or needed improving.

\section{The Program Intervention}

Participants received pre and post cognitive and health screening services from the RN running the program; were provided with an iPad and activity tracker (Misfit) each, then given an eight-week group training program using electronic tablets (iPads) that focused on addressing risk factors for dementia, and a further 1 month of self-directed goal-focused activity using the iPad and Misfit activity tracker. Post data collection was carried out three months after commencing the program.

The other aspects of the evaluation relied on interview data. Interviews with thirteen participants were carried out approximately four weeks after the program finished along with interviews with the nurses delivering the program. Questions were based on understanding participants and RN's experiences of the key aspects of the program - group work, goal setting, technology use, social connection and behaviour change to decrease dementia risk factors.

\section{Results}

Reach

The mean age of participants was 76.05 years (range 53-94), 76.5\% were female and $45.9 \%$ were married, with over a third to half of participants having a medical history of arthritis, hypertension or heart disease (See Table 1) demonstrating a good reach of the program to older people with chronic illness. The MWP was delivered across 18 rural and urban sites within Australia, demonstrating delivery flexibility and adaptability.

\section{Table 1: Tasmanian Memory Wellness Program Participant characteristics}

\section{Effectiveness}

As displayed in Table 2, there are statistically significant differences, at the .05 significance level, in pretest to post-test scores for ACE-III, Diastolic BP, weight, stress and TIU5 (have you been on the internet for any reason besides email and texting). Results show that cognition increased, diastolic BP decreased, weight increased, stress decreased and TIU5 decreased (which equalled an increase in use) after exposure to the memory wellness program.

Cognition: At pre-test the mean ACE-III for 89 participants was 88.43 increasing at post-test to 90.72 , which was significant at the 0.05 level (moderate to large effect size of $r 0.88$ ).

Most clinical data did not register statistically significant changes including BGL, BMI, and the DASS measures for depression, or anxiety. It appears that for some participants the nurse-led clinic components 
led to referral for other problems. 'Since I've been in this program, the memory wellness, I've discovered that I've got the onset of diabetes. I probably need to focus a bit more on that so I don't get any worse. I don't need medication at the moment and [nurse's name] showed me that my blood sugar levels have gone down a bit so it's a good start'(HB17).

Interviewees' main motivations for undertaking the program were to improve memory, meet new people and improve technology use and computer literacy, and the program provided these elements for most participants. Well we got the invitation and I thought it would be a good idea, to you know keep my memory going better. And l've actually made some improvement. From the first assessment to this one, I've improved. (HB04). Many of the interview respondents indicated that they now tried to use their memory or learn new things more. 'Yes [doing] something new that you haven't done before. That's what I got out of the course.'(LA18).

Physical Activity: There was little shift in participants 'stage of change' for physical activity data, with most participants remaining in the stage they initially identified (ranging from contemplating change to post-change stage). At interview, a few specifically reported increasing their exercise and that the Misfit activity tracker provided motivation to exercise more. The misfit, I think it's a wonderful little gadget. Because I've always walked, now I can know [how far] I actually walk and make goals and I can make sure I do my things. I'm on a 56 day streak (L 19).

Technology: The participants were mostly already actively using technology and the internet, meaning our TIU tool was not sensitive enough to measure any change from already active users. The interviews provided further context to the impact of the program on participants' use of technology. Reported improvements in technology use were variable. Some found it relatively simple to use both the Misfit activity tracker and the iPad due to past experience, already possessing similar items, or having a general interest in technology. Yes, I referred to it [Misfit Activity Tracker] a lot. I don't know how accurate it is. At least it tells me a story. And my wife downloaded the app onto my phone so I get all the information from there onto my phone, and I check that every day or two (HB17). Some tracked their fitness regularly as a result of the tracker, some had issues with syncing the device to their iPad, while others gave up on using it early on in the program. Interestingly, some participants reported that they were making conscious decisions to undertake more daily exercise, even though they were unsure of how to use the tracker. For a few, they expanded their use of technology, undertaking new activities such as taking photographs or playing memory games. Some participants reported an increase in their use of technology during the program, especially those who had not owned an iPad previously and who found the games and puzzles useful. Well, oh well I love it [ipad]. I'd never touched anything like that...I do that letter soup everytime, I love it... (HB05). Others however, reported difficulties with using the iPad or were not interested in the brain puzzles. Therefore, it seems that the outcomes from incorporating technology into the program were very person-dependent, and the program benefits from a multi-pronged approach.

Social Interaction: Our measure of loneliness ULS- 6 demonstrated a trend towards a decrease in feelings of loneliness. While not statistically significant, the pre-test result (mean 11.49) closely matched the ULS- 
6 data of over 1,000 older adults in Portugal (25), unlike the post-test data (mean 10.77) which closely matched the married cohort of Portuguese older adults (25), suggesting a positive downward trend in loneliness. The interviews support this interpretation, as meeting and interacting with others was given as the primary positive element of the program. Participants reported enjoying sharing their stories, discussing what they were learning, helping one another and laughing over their difficulties with mastering technology. Oh I think, well naturally the company [was something I enjoyed]. I think everybody does. And the thing is, we were bouncing things of each other, which made you think.(LA08). Two interviewees indicated that loneliness prior to the program had been a key issue that the MWP helped resolve. '...but it took so much to make myself get here. It got easier and I've made friends and it's lovely, I look forward to it (HB05). Overall, most participants reported low levels of loneliness at pre-test, limiting the capacity of the intervention to improve loneliness, and future research should consider a more sensitive socialisation measure.

\section{Adoption}

Some participants indicated that they had adopted some of the risk reduction strategies taught during the program But I'm a lot fitter now that I was. After we finished here [at MWP] each week we used to go over to the other side of the river and do exercises for an hour and a half (HO3). But different responses to the program were indicated from each individual interviewed, suggesting that the program variety meant there was at least one thing that could be taken up by attendees. Attendance to the program was good for about two thirds of respondents, with a third of participants not having the final assessment.

\section{Implementation}

The data collection and interviews highlighted gaps in the program implementation. Whilst goal setting was stated as one of the main features of the program, there were no goals captured for the data collection. At interview some participants did recall setting specific goals, including doing more activities to improve memory, improving their diet, doing more exercise, becoming more informed about brain health and socialising more.

The technology elements of the program are a positive and integral element of the program. However, the provision of iPads was not required by all participants as some were already very actively using their own iPads or tablets. A better goal setting process could have identified participants requiring greater support with technology, with potential cost benefits for the MWP and the ability to allocate additional time to those who need more support to use iPads - a few participants were unable to 'get going' with the iPad technology and gave up trying. The presence of support workers for assisting with technology was appreciated by some interviewees.

The education sessions appear to have raised awareness for participants, according to the interview data. Feedback from some respondents recommended training for RNs in group education, to ensure that the sessions were more engaging and inclusive, particularly for participants who were quiet or had hearing difficulties. Some also said they would appreciate the inclusion of additional activities between the formal presentations including relaxation/stress management techniques, memory games and social 
elements to increase the value of the sessions. Some participants organised their own activities between sessions. Some participants reported that the initial sessions were quite crowded and chaotic with many confused about what they were supposed to be doing. For some groups, the venues were too small and the number of participants attending on the first day were too large for the room. While groups were often subsequently split the first session felt crowded and disorganised. Some participants though, went on to enrol in other similar integratedliving health promotion programs, suggesting an overall enjoyment and benefit for them.

The evaluation uncovered elements of the program that could be improved, but it must be noted that the delivery of the program in Tasmania in 2017 was impacted by changes in the staff delivering the programs, so that some participants worked with 3 nurses over the life of their program.

\section{Maintenance}

Management from integratedliving have provided strong support to the continuation of the program reporting that steps have already been taken to address the results of the evaluation. The program has ongoing funding.

\section{Discussion And Implications}

Our findings support other multi-modal studies (5) on the benefits of mixed interventions. Our qualitative results suggest that the multi-modal design providing both choice and multiple behaviour change techniques was pivotal to improved cognitive scores as participants took up activities from at least some element of the program, but none adopted all recommended behaviours. The success then of a multimodal program may be based on providing participants with goal and intervention choices, not on adoption of a full range of behavioural changes. This factor may also explain the differences in findings related to the varied evidence on success of multi-modal programs (30), where a strong focus on a goal of relevance provides stronger intention for changing health behaviour. This aspect of the MWP could not be analysed because the goal setting activity was not routinely documented, but we suggest goal setting analysis be added to future multi-modal health promotion research.

There is a need to develop more sensitive research tools to study technology use in the older population. Most of our participants already used technology, meaning the TIU tool (adapted from 26)) lacked sensitivity to detect any change in use whether that be frequency or application. Qualitative data suggests that the iPad and Misfit activity tracker aspects of the program did encourage and increase most participants' engagement through learning new technology and/or new online activities. As well, increases in levels of physical activity were reported by a few interviewees but no shifting in the stages of changes using the PA-SoC tool (27). It is possible that any increase in activity occurred for those already in the action or maintenance stages of change, and we suggest further work to measure and incorporate this theoretical approach into real-world evaluations. 
Both the decrease in loneliness and the qualitative accolades for the social interaction elements of the program, suggest the group work is a key factor in the program's outcomes. These results support the findings that social networks and social engagement are negatively associated with cognitive decline (12, 13), and while our data do not link internet based social media as a form of social interaction these could be fruitful avenues for improvements in cognition (15).

The improvement in cognition (ACE-III) is promising but further research is required to confirm the results. This real-world evaluation had numerous limitations that deserve consideration. The gaps in data collection meant that the sample is small for our technology, loneliness, and physical activity stages of change measures which limited power in assessing change. The real-world setting for data collection inevitably raises questions about the quality of the data gathered, though the changes noted were similar across 18 sites suggesting, a real effect. The design did not attempt to isolate any particular activity or process as being causal in changes, and therefore did not measure dose or fidelity, instead relying on the overall program synergy as others have done (8). Many dementia related studies now identify the risks of practice effects, where cognitive scores improve by as much as 0.25 through repeated applications of tests (31). AceMobile provides several versions of the test to mitigate this risk, but there is no record of whether different versions were or were not used by RNs. However, given that practice effects may last several years (32), that we do not know whether participants were also receiving cognitive testing through GPs or other sources, and that our effect sizes were moderate to large (total ACE .578 cohens d, attention .868 , memory .566 , language 0.4 , visuospatial 0.595 ) and consistent across 18 sites, any practice effects will not account for the full effect size.

A strength of this study is that it evaluated a real-world program based on translating evidence into practice. While the evaluation has limitations caused by the real-world problems of gaps in program delivery and data collection, staff changes, and client drop outs, it also enrolled 'real populations' with participants in older age ranges rarely studied and assessed implementation across 18 sites (22). The improvement in diastolic blood pressure, along with the non-statistical improvement in loneliness, alongside the qualitative reports of program enjoyment demonstrate that the MWP provided benefits to participants.

The evaluation did not attempt to identify dose related changes, but rather worked on the premise that the multi-modal MWP provided cognitive and social stimulation using a range of options and behaviour change techniques. The expectations was that participants would select some (not all) activities that felt relevant to them for healthy brain function in their lives, such as using their memory, improving their diet, incorporating exercise, doing brain puzzles and games, and learning new activities. Like Brayne and Davis (22) we suggest that rather than research resources being focused on the impact of reducing individual risk factors and the extent of change needed, that assessing the impact of overall lifestyle programs that can be adopted by older people and that delay the onset or trajectory of dementia in the older population, are likely to achieve the greatest benefit. Further research is needed to clarify whether these results are reproducible and sustainable. 


\section{Declarations}

Ethics approval was granted for this study by the Human Research Ethics Committee (Tasmania) Network: Reference H0015530.

The datasets used and/or analysed during the current study are available from the corresponding author on reasonable request.

Author IA is an employee of integratedliving and all other authors declare they have no competing interests.

This work was supported with grant assistance from the Tasmanian Community Fund Board [grant number S24717/CNT06995]. The grant was awarded based on a competitive funding application, with no subsequent involvement in the project.

Authors contributions: CS, HM and CK designed the study. CS and AT collected and analysed the data with assistance from CK and IA. CS drafted the paper and all authors read and approved the final manuscript.

\section{References}

1. Orrell M, Brayne C. Dementia prevention: call to action. The Lancet. 2015;386(10004):1625.

2. World Health Organization. Risk reduction of cognitive decline and dementia: WHO guidelines. Geneva: World Health Organization; 2019.

3. Baumgart M, Snyder HM, Carrillo MC, Fazio S, Kim H, Johns H. Summary of the evidence on modifiable risk factors for cognitive decline and dementia: A population-based perspective. Alzheimer's \& Dementia. 2015;11(6):718-26.

4. Olanrewaju O, Clare L, Barnes L, Brayne C. A multimodal approach to dementia prevention: A report from the Cambridge Institute of Public Health. Alzheimer's \& Dementia: Translational Research \& Clinical Interventions. 2015;1(3):151-6.

5. Ngandu T, Lehtisalo J, Solomon A, Levälahti E, Ahtiluoto S, Antikainen R, et al. Articles: A 2 year multidomain intervention of diet, exercise, cognitive training, and vascular risk monitoring versus control to prevent cognitive decline in at-risk elderly people (FINGER): a randomised controlled trial. The Lancet. 2015;385:2255-63.

6. Bherer L, Erickson $\mathrm{KI}$, Liu-Ambrose T. A review of the effects of physical activity and exercise on cognitive and brain functions in older adults. J Aging Res. 2013;2013:657508.

7. Devore EE, Kang JH, Breteler MM, Grodstein F. Dietary intakes of berries and flavonoids in relation to cognitive decline. Annals of neurology. 2012;72(1):135-43. 
8. Carlson MC, Saczynski JS, Rebok GW, Seeman T, Glass TA, McGill S, et al. Exploring the Effects of an "Everyday" Activity Program on Executive Function and Memory in Older Adults: Experience Corps $\AA^{\circledR}$. The Gerontologist. 2008;48(6):793-801.

9. Dannhauser TM, Cleverley M, Whitfield TJ, Fletcher B, Stevens T, Walker Z. A complex multimodal activity intervention to reduce the risk of dementia in mild cognitive impairment-ThinkingFit: pilot and feasibility study for a randomized controlled trial. BMC Psychiatry. 2014;14(1):129.

10. Horning SM, Young S, Myhre JW, Osato S, Wilkins SS. A Multimodal Cognitive Enhancement Program for Older Adults: A Case Report of the Implementation of Brain Training. Activities, Adaptation \& Aging. 2016;40(4):320-38.

11. Kim K, Han JW, So Y, Seo J, Kim YJ, Park JH, et al. Cognitive Stimulation as a Therapeutic Modality for Dementia: A Meta-Analysis. Psychiatry investigation. 2017;14(5):626-39.

12. Barnes LL, Mendes de Leon CF, Wilson RS, Bienias JL, Evans DA. Social resources and cognitive decline in a population of older African Americans and whites. Neurology. 2004;63(12):2322-6.

13. James BD, Wilson RS, Barnes LL, Bennett DA. Late-Life Social Activity and Cognitive Decline in Old Age. Journal of the International Neuropsychological Society. 2011;17(6):998-1005.

14. Holwerda TJ, Deeg DJ, Beekman AT, van Tilburg TG, Stek ML, Jonker C, et al. Feelings of loneliness, but not social isolation, predict dementia onset: results from the Amsterdam Study of the Elderly (AMSTEL). Journal of neurology, neurosurgery, and psychiatry. 2014;85(2):135-42.

15. Myhre JW, Mehl MR, Glisky EL. Cognitive Benefits of Online Social Networking for Healthy Older Adults. The journals of gerontology Series B, Psychological sciences and social sciences. 2017;72(5):75260 .

16. Strecher VJ, Seijts GH, Kok GJ, Latham GP, Glasgow R, DeVellis B, et al. Goal setting as a strategy for health behavior change. Health education quarterly. 1995;22(2):190-200.

17. Epton T, Currie S, Armitage CJ. Unique effects of setting goals on behavior change: Systematic review and meta-analysis. Journal of consulting and clinical psychology. 2017;85(12):1182-98.

18. McEwan D, Harden SM, Zumbo BD, Sylvester BD, Kaulius M, Ruissen GR, et al. The effectiveness of multi-component goal setting interventions for changing physical activity behaviour: a systematic review and meta-analysis. Health psychology review. 2016;10(1):67-88.

19. Stephenson A, McDonough SM, Murphy MH, Nugent CD, Mair JL. Using computer, mobile and wearable technology enhanced interventions to reduce sedentary behaviour: a systematic review and meta-analysis. The international journal of behavioral nutrition and physical activity. 2017;14(1):105. 
20. Conner M, Abraham C, Prestwich A, Hutter R, Hallam J, Sykes-Muskett B, et al. Impact of goal priority and goal conflict on the intention-health-behavior relationship: Tests on physical activity and other health behaviors. Health psychology : official journal of the Division of Health Psychology, American Psychological Association. 2016;35(9):1017-26.

21. Green LW, Ottoson JM, García C, Hiatt RA. Diffusion Theory and Knowledge Dissemination, Utilization, and Integration in Public Health. Annual Review of Public Health. 2009;30(1):151-74.

22. Brayne C, Davis D. Making Alzheimer's and dementia research fit for populations. The Lancet. 2012;380(9851):1441-3.

23. Strandberg TE, O'Neill D. Dementia : a geriatric syndrome. The Lancet. 2013;381(9866):533-4.

24. Glasgow RE, Vogt TM, Boles SM. Evaluating the Public Health Impact of Health Promotion Interventions: The RE-AIM Framework. American Journal of Public Health. 1999;89(9):1322-7.

25. Neto F. Psychometric analysis of the short-form UCLA Loneliness Scale (ULS-6) in older adults. Eur J Ageing. 2014;11(4):313-9.

26. Choi NG, Dinitto DM. Internet use among older adults: association with health needs, psychological capital, and social capital. Journal of medical Internet research. 2013;15(5):e97.

27. Marcus BH, Forsyth LH. Motivating People to Be Physically Active: Champaign; 2003 [Available from: https://www.edu.gov.mb.ca/k12/cur/physhlth/frame_found_gr11/rm/1_fm.pdf.

28. So M, Foxe D, Kumfor F, Murray C, Hsieh S, Savage G, et al. Addenbrooke's Cognitive Examination III: Psychometric Characteristics and Relations to Functional Ability in Dementia. Journal of the International Neuropsychological Society. 2018;24(8):854-63.

29. Newman CGJ, Bevins AD, Zajicek JP, Hodges JR, Vuillermoz E, Dickenson JM, et al. Improving the quality of cognitive screening assessments: ACEmobile, an iPad-based version of the Addenbrooke's Cognitive Examination-III. Alzheimer's \& Dementia: Diagnosis, Assessment \& Disease Monitoring. 2018;10:182-7.

30. Conner M, Norman P. Health behaviour: Current issues and challenges. Psychology \& health. 2017;32(8):895-906.

31. Goldberg TE, Harvey PD, Wesnes KA, Snyder PJ, Schneider LS. Practice effects due to serial cognitive assessment: Implications for preclinical Alzheimer's disease randomized controlled trials. Alzheimers Dement (Amst). 2015;1(1):103-11.

32. Mathews M, Abner E, Caban-Holt A, Kryscio R, Schmitt F. CERAD practice effects and attrition bias in a dementia prevention trial. International psychogeriatrics. 2013;25(7):1115-23. 


\section{Tables}

Table 1: Tasmanian Memory Wellness Program Participant characteristics

\begin{tabular}{l|l|rrrr} 
& & $\begin{array}{c}\text { No } \\
\text { count }\end{array}$ & $\begin{array}{r}\text { Yes } \\
\text { count }\end{array}$ & \% Total Count \\
\hline Sex & Female & 130 & 76.5 & \\
\hline Age & Male & 40 & 23.5 & 170 \\
\hline & Mean/Range & 76.05 & $(53-94)$ & 159 \\
\hline
\end{tabular}

\begin{tabular}{l|rr}
\hline Marital Status (TAS data only Married & 16 & 19.8
\end{tabular}

\begin{tabular}{l|rr}
\hline Single & 6 & 7.4 \\
\hline Widowed & 13 & 16.0
\end{tabular}

\begin{tabular}{|c|c|c|c|c|c|}
\hline & N/A & 1 & 1.2 & & 36 \\
\hline \multirow{9}{*}{$\begin{array}{l}\text { Medical History } \\
\text { TAS } \\
\text { Data only }\end{array}$} & Hypertension & 18 & 54.5 & 1545.5 & 33 \\
\hline & Heart disease & 20 & 60.6 & 1339.4 & 33 \\
\hline & Arthritis & 12 & 36.4 & 2163.6 & 33 \\
\hline & Diabetes & 26 & 78.8 & 721.2 & 33 \\
\hline & Lung disease & 33 & 100.0 & 00.0 & 33 \\
\hline & Dementia & 32 & 97.0 & 13.0 & 33 \\
\hline & Cancer & 26 & 78.8 & 721.2 & 33 \\
\hline & CVA & 30 & 90.9 & 39.1 & 33 \\
\hline & Mental Illness & 27 & 81.8 & 618.2 & 33 \\
\hline
\end{tabular}

Table 2: Paired sample t-test results for Memory Wellness Program 


\begin{tabular}{|c|c|c|c|c|c|c|c|c|c|}
\hline \multirow[b]{2}{*}{ Outcome } & \multicolumn{2}{|c|}{ Pretest } & \multicolumn{2}{|c|}{ Posttest } & & \multirow[t]{2}{*}{ 95\% Cl for Mean Difference } & \multirow[b]{2}{*}{ I } & \multirow[b]{2}{*}{$\mathbf{t}$} & \multirow[b]{2}{*}{ df } \\
\hline & M & SD & M & SD & $\mathbf{N}$ & & & & \\
\hline Acelll - cognition & 88.43 & 7.04 & 90.72 & 7.42 & 89 & $-3.04,-1.54$ & $0.88 *$ & -6.07 & 88 \\
\hline Heart_Rate & 71.93 & 9.34 & 71.48 & 10.37 & 40 & $-2.42,3.32$ & 0.59 & .317 & 39 \\
\hline Systolic_BP & 144.48 & 25.63 & 135.15 & 18.94 & 62 & $3.61,15.07$ & $0.52 *$ & 3.23 & 61 \\
\hline Diastolic_BP & 82.24 & 16.69 & 78.79 & 9.86 & 62 & $-.63,7.53$ & 0.36 & 1.69 & 61 \\
\hline BGL & 7.81 & 2.21 & 7.75 & 2.43 & 49 & $-.70, .83$ & 0.34 & .17 & 48 \\
\hline Weight & 79.49 & 15.68 & 84.30 & 16.58 & 33 & $-8.38,-1.24$ & $0.81 *$ & -2.74 & 32 \\
\hline DASS Depression & 4.07 & 3.42 & 3.93 & 3.54 & 56 & $-7.67,1.05$ & 0.52 & .32 & 55 \\
\hline DASS Anxiety & 3.40 & 3.21 & 3.36 & 3.25 & 55 & $-.82, .90$ & 0.52 & .09 & 54 \\
\hline Stress & 5.15 & 3.45 & 4.06 & 3.15 & 55 & $.27,1.92$ & $0 . .58 *$ & 2.65 & 54 \\
\hline Ucla & 11.49 & 3.83 & 10.77 & 3.90 & 35 & $-.32,1.75$ & 0.70 & 1.40 & 34 \\
\hline Stage (PA-SoC) & 3.60 & 1.33 & 3.60 & 1.22 & 35 & $-.33, .33$ & 0.71 & .00 & 34 \\
\hline ACEIII Attention & 15.57 & 1.63 & 16.89 & 1.43 & 34 & $-1.87,-.78$ & $.49 *$ & -4.95 & 33 \\
\hline ACEIII Memory & 22.42 & 3.36 & 24.21 & 2.95 & 34 & $-3.12,-.45$ & $.27^{*}$ & -2.73 & 33 \\
\hline ACEIII Fluency & 10.04 & 2.49 & 10.06 & 2.26 & 34 & $-.64, .59$ & .72 & -.08 & 33 \\
\hline ACEIII Language & 23.92 & 4.22 & 25.22 & 1.703 & 34 & $-2.76, .16$ & .21 & -1.81 & 33 \\
\hline ACE Visuospacial & 12.81 & 1.96 & 13.96 & 1.88 & 34 & $-1.88,-.42$ & $.41 *$ & -3.21 & 32 \\
\hline
\end{tabular}

*P value $<0.05$ 